\title{
Une politique de développement de l'interdisciplinarité : l'action du département des Sciences humaines et sociales du ministère de la Recherche (2002-2004)
}

\author{
Marcel Jollivet \\ Sociologue, Université Paris X, CNRS, LADYSS, Bât. K, 200 avenue de la République, 92001 Nanterre cedex, France
}

Dans le but de développer l'interdisciplinarité au sein, et autour, des sciences humaines et sociales, ce département ministériel a pris de multiples initiatives de différentes natures (Encadré 1). Cette intense activité mérite en soi d'être signalée ${ }^{1}$. De plus, elle présente sur le fond un double intérêt. En premier lieu, tant par le contenu des appels à propositions que par l'ampleur de la mobilisation qu'ils ont provoquée (plus de six cents réponses au total), elle constitue une véritable enquête sur la place et le sens actuels, en France, de l'interdisciplinarité dans le secteur des sciences humaines et sociales, autour des thématiques retenues. Celles-ci couvrent, soulignons-le d'entrée, un champ d'investigation qui déborde largement la seule compétence de ces disciplines et exigent donc une interdisciplinarité qui les déborde aussi. Les thèmes de recherche privilégiés expriment les besoins de connaissance qui justifient cet appel, tout à fait inhabituel. Les projets que les équipes soumettent, de leur côté, renseignent sur les besoins qu'elles ressentent de cette interdisciplinarité et sur la pratique qu'elles s'estiment en mesure d'en avoir. Voici donc une occasion unique en son genre de dresser un état des lieux, au moins tel qu'il apparaît à travers ces réponses.

En second lieu, il est tout particulièrement intéressant de faire la part, dans l'ensemble des thématiques qui sont mises en avant, de celles portant sur les questions d'environnement, de développement durable, de risque, de santé, ainsi que sur les problèmes méthodologiques que pose l'interdisciplinarité requise pour en traiter. On verra en effet que leur place est importante, ce qui est tout à fait significatif en soi. Cela permet en outre de se donner une idée des démarches interdisciplinaires proposées par les équipes pour les aborder.

Auteur correspondant : marcel.jollivet@u-paris10.fr

${ }^{1}$ La liste de l'encadré 1 ne prétend pas à l'exhaustivité. On pourrait y inclure les actions concernant la «ville durable».

\section{Des appels à propositions placés sous le signe du rapprochement des disciplines}

Un seul des appels à propositions (celui de l'action thématique concertée [ATC] «Environnement et santé ») ne fait aucune référence à une interdisciplinarité souhaitée. Présent dans toutes les autres actions, cet appel y est toutefois plus ou moins pressant. C'est dans les attendus de l'action concertée incitative (ACI) « Terrains, techniques, théories » qu'il est le plus explicite. «L'objectif majeur est donc de promouvoir l'interdisciplinarité », y est-il clairement annoncé. On doit noter le souci d'ouverture au-delà des sciences sociales qui est affiché dans certaines des actions : référence à une interdisciplinarité entre les sciences sociales et les «autres sciences » dans l'ACI «Terrains, techniques, théories », à des collaborations entre les sciences sociales et les «sciences de la matière et du vivant » dans l'ACI « Sociétés et cultures dans le développement durable», à des collaborations «avec les chercheurs des sciences physiques et des sciences de la vie » à propos du séminaire «Environnement et développement : quelles questions pour la recherche en sciences humaines et sociales? ». Enfin, les disciplines des sciences de la Terre contribuant à la recherche sur l'évolution du climat et celles qui s'intéressent aux conséquences des changements climatiques sur les systèmes productifs (agricoles, alimentaires et industriels) étaient présentes dans le séminaire "Changements globaux : vulnérabilité, adaptabilité, apprentissage ».

Le souci de faire se confronter, voire de faire travailler ensemble, à tout le moins différentes sciences humaines et sociales et, au-delà parfois, sciences sociales et sciences physiques ou biologiques, est donc affirmé comme un objectif prioritaire. Mais de quelle forme d'interdisciplinarité s'agit-il ? Les appels à propositions fournissent-ils des 
Encadré 1. Liste des actions interdisciplinaires du département Sciences humaines et sociales de la Direction de la recherche du ministère délégué à la Recherche et aux Nouvelles Technologies (2002-2004)

- Action concertée incitative (ACI) «Terrains, techniques, théories. Travail interdisciplinaire en sciences sociales » (2002-2004) 4 appels à propositions (2 en 2002, 1 en 2003, 1 en 2004)

- ACI «Sociétés et cultures dans le développement durable» (2003-2004) : 2 appels à propositions (1 en 2003, 1 en 2004). Cet appel d'offres est lancé par le ministère en collaboration avec l'Inra et l'IRD

- ACI « Normes, pratiques et régulations des politiques publiques » (2004) : 1 appel à propositions

- Action thématique concertée (ATC) « Environnement et santé » (2004) : 1 appel à propositions

- Séminaire « Environnement et développement : quelles questions pour la recherche en sciences humaines et sociales ?» (2002) :

5 journées (21 mars, 18 avril, 16 mai, 13 juin, 27 juin)

- Séminaire «Changements globaux : vulnérabilité, adaptabilité, apprentissage » (10 décembre 2003). Ce séminaire était organisé avec le concours du Programme international sur les dimensions humaines du changement global (International Human Dimensions Programme on Global Environmental Change, IHDP), de l'INSU (CNRS), du programme GICC, de l'Inra, de l'IRD et de l'INED.

indications qui permettent de lui donner un contenu ou au moins d'aller dans ce sens? Par exemple, très simplement, suggèrent-ils des procédures susceptibles d'induire une pratique du travail interdisciplinaire et sur la base desquelles il pourra être procédé à l'évaluation des propositions et au choix de celles qui seront retenues?

\section{Du côté de l'offre, quelles problématiques et quelles modalités pour quelle interdisciplinarité ?}

Si on examine les appels à propositions sous cet angle, seul celui de l'ACI «Terrains, techniques, théories » retient véritablement l'attention. L'exigence première d'un véritable travail interdisciplinaire en est le fondement même. Elle est énoncée en une phrase lapidaire dans les attendus. «Le travail interdisciplinaire, est-il dit, exige [...] un effort soutenu de réflexivité sur les pratiques cognitives et les méthodes des disciplines. » Cette phrase va à l'essentiel. Et ce qui en découle également. Ainsi «l'objectif » déclaré de l'ACI est-il de « rendre intelligibles les multiples procédures qui permettent [aux sciences humaines et sociales] de construire et de faire évoluer, dans une relation permanente aux autres disciplines, leurs terrains, leurs techniques, leurs théories ». Voilà bien en effet la voie de $l^{\prime}$ «interconnaissance » et de la compréhension réciproque qui est la condition initiale pour qu'un échange fructueux, sans compromis hasardeux ni malentendus, se produise entre les chercheurs des différentes disciplines.

Il est aussi significatif que la voie des «spéculations abstraites » soit écartée au profit de confrontations sur la base de « recherches précises sur des thèmes bien identifiés ». L'inspiration est judicieusement pragmatique. "C'est souvent via les terrains et les techniques, est-il souligné, que s'élaborent les démarches interdisciplinaires ; ceux-ci nécessitent des procédures d'ajustement entre les disciplines souvent plus aisées et moins chargées de significations institutionnelles et symboliques que ce que réclame l'ajustement des points de vue sur les théories ; enfin, les interdépendances sont souvent fortes entre les formes du travail de terrain, les techniques d'une discipline et les théories qu'elle construit. » Il s'agit donc bien d'expérimenter des pratiques interdisciplinaires et de le faire sur la base d'une explicitation partagée des principes des pratiques disciplinaires.

D'où certaines attentes et certaines contraintes, à destination des projets, mais aussi des possibilités originales qui leur sont offertes. Parmi les attentes, c'est tout d'abord qu'il soit procédé à un approfondissement des notions de «terrain", de «technique» et de «théorie», c'est le souhait d'un centrage des thématiques de recherche sur les articulations qui existent entre ces trois termes. Du côté des contraintes, c'est celle, clairement énoncée, pour les projets de recherche, d'associer au moins deux disciplines. Quant aux possibilités offertes, elles consistent en modalités de travail particulièrement élaborées pour permettre le travail de fond qu'appelle l'interdisciplinarité. Tout d'abord, à côté du classique projet de recherche, place est faite à la technique, elle aussi bien connue, de la « déclaration d'intention » permettant de demander à pouvoir bénéficier d'une période de travail nécessaire à la mise en place effective d'un programme scientifique. Jusquelà, rien de très original. Mais l'ACI ouvre en plus la possibilité de créer également des "structures d'échanges et de recherche partenariales » de formes diverses ${ }^{2}$. La diversité et la souplesse de ces formules traduisent le souci qu'a l'ACI d'adapter son action aux contraintes du découpage disciplinaire des laboratoires et aux difficultés d'une tâche pionnière. À travers toutes ces formules, apparaît également le souci - primordial - de donner du

\footnotetext{
${ }^{2}$ Les formules proposées sont multiples : des « réseaux de recherche, groupements destinés à mettre en commun des recherches en cours", des "ateliers de recherche exploratoire, destinés à lancer une réflexion, à partir de projets de recherche en cours de définition, sur une problématique nouvelle, impliquant un travail interdisciplinaire qui pose des problèmes de compréhension entre disciplines », des "écoles thématiques » visant à «dynamiser la réflexion collective et interdisciplinaire sur les utilisations comparées d'une ou de plusieurs techniques $» .$.
} 


\begin{abstract}
Encadré 2. Les grands thèmes de recherche (ACI/ATC)
«Terrains, techniques, théories» : «De l'icône au visuel: l'image en sciences humaines et sociales», «Approches de la complexité », «Identité(s) », «Monde(s), mondialisation », «Règles, normes, régularités », « Le présent », « Mesures, données : catégorisation, agrégation, modélisation, numérisation, diffusion », « Le naturel et l'artificiel », « La peur et les risques », « Âges, temps de vie et rapport de génération », « Pratiques et techniques du corps », « Traduire, interpréter, transmettre », «L'erreur ».

Thème permanent : SHS des SHS, dynamiques des méthodes, des objets, des enjeux dans les disciplines des sciences humaines et sociales.

«Sociétés et cultures dans le développement durable» : «Cultures, pratiques sociales et temporalités », «De nouvelles recherches sur le développement », «Gouvernement des sociétés et action collective », "Concevoir, produire, consommer, vivre ensemble», «La durabilité en question », "Vulnérabilité et adaptabilité », "Dynamique des systèmes de production : de la conception à la consommation ».

« Normes, pratiques et régulations des politiques publiques»: «Les formes de l'action publique : rupture et continuité », « Les acteurs et les institutions », «Entre normes et régulations? ».

«Environnement et santé » : «Nouveaux instruments : données, méthodes, indicateurs », "Connaissance des populations exposées et des zones à risques : les disparités socio-spatiales de santé», «Les pratiques et les représentations sociales; Les modes de vie », « Le rôle des acteurs et des institutions ; La production des normes ; Les modes d'action ».
\end{abstract}

temps à des entreprises dont on a bien conscience qu'elles en exigent.

Les intitulés des thèmes sur lesquels cette démarche interdisciplinaire est invitée à s'appliquer sont en complète cohérence, par leur ampleur et leurs dimensions transversales aux disciplines, avec le propos interdisciplinaire de l'ACI. On peut parler de thèmes « complexes » (Encadré 2).

La priorité accordée à des thèmes de caractère explicitement méthodologique, tels que «Approches de la complexité », "Mesures, données : catégorisation, agrégation, modélisation, numérisation, diffusion » et «L'erreur ", tout à fait exceptionnelle dans ce genre d'appel, va dans le même sens. Ces thèmes méritent ici une attention particulière dans la mesure où, placés comme ils le sont dans des appels à propositions centrés sur l'interdisciplinarité, ils sont censés aborder les problèmes méthodologiques de celle-ci. C'est aussi le cas du thème permanent, repris dans les appels à propositions successifs, concernant les sciences humaines et sociales (Encadré 2 ) et qui illustre à soi seul l'ambition interdisciplinaire de l'ACI et l'esprit dans lequel elle est conçue. On y retrouve en effet le souci d'autoréflexivité disciplinaire qui doit présider à la mise en commun interdisciplinaire. Avec cette ACI, on a donc bien affaire à une incitation clairement ciblée sur l'interdisciplinarité. Les précisions apportées sont autant d'indications de distance prise avec la simple pluridisciplinarité. En outre, l'on ne peut qu'être frappé par la pertinence des questions méthodologiques soulevées : cette $\mathrm{ACI}$ est à l'évidence le fruit d'une réelle réflexion.

L'ouverture interdisciplinaire des autres actions est beaucoup plus limitée. Elle se réduit au fait que leurs thématiques et leurs problématiques s'adressent à une gamme très étendue de disciplines (pas seulement, on l'a vu, de sciences humaines et sociales). L'intérêt de ces textes est de balayer le champ des questions actuelles dans les domaines ciblés par les ACI et de les présenter d'une façon qui ne les segmente pas en compartiments disciplinaires. Mais, du point de vue de la mise en œuvre de l'interdisciplinarité (ou même de la simple pluridisciplinarité), on en reste à l'exhortation initiale invitant les disciplines à coopérer. Seule indication novatrice : il est à plusieurs reprises précisé qu'il faut réfléchir «aux modalités de la mise en œuvre du travail interdisciplinaire ». Cet accent mis sur la méthode - point crucial de l'interdisciplinarité - mérite d'être souligné. Mais aucune piste n'est fournie, ni même esquissée, pour montrer comment tel ou tel des thèmes proposés à l'analyse gagnerait à être pris en charge simultanément de plusieurs points de vue disciplinaires. À cette discrétion, une seule exception, importante, il est vrai : c'est lorsqu'il est fait référence à la nécessité de confronter les présupposés et les principes des démarches des disciplines, en vue d'une construction partagée d'un champ de recherche intégrant environnement et développement.

Voici donc une occasion offerte de s'attaquer aux problèmes de fond de l'interdisciplinarité avec les moyens de les affronter. A-t-elle été saisie? Et comment?

\section{Les réponses des équipes : quelle(s) interdisciplinarité(s) affichée(s)? Avec quels objectifs?}

Les listes des projets reçus ${ }^{3}$ en réponse à l'ensemble des appels à propositions se prêtent à deux formes d'exploitation. Elles permettent en premier lieu de dresser

\footnotetext{
${ }^{3}$ Les analyses qui suivent sont faites à partir des listes des projets reçus. Celles-ci fournissent la composition des équipes avec les disciplines des chercheurs, ainsi que le titre du projet et sa classification dans les mots-clés de l'appel à propositions concerné.
} 


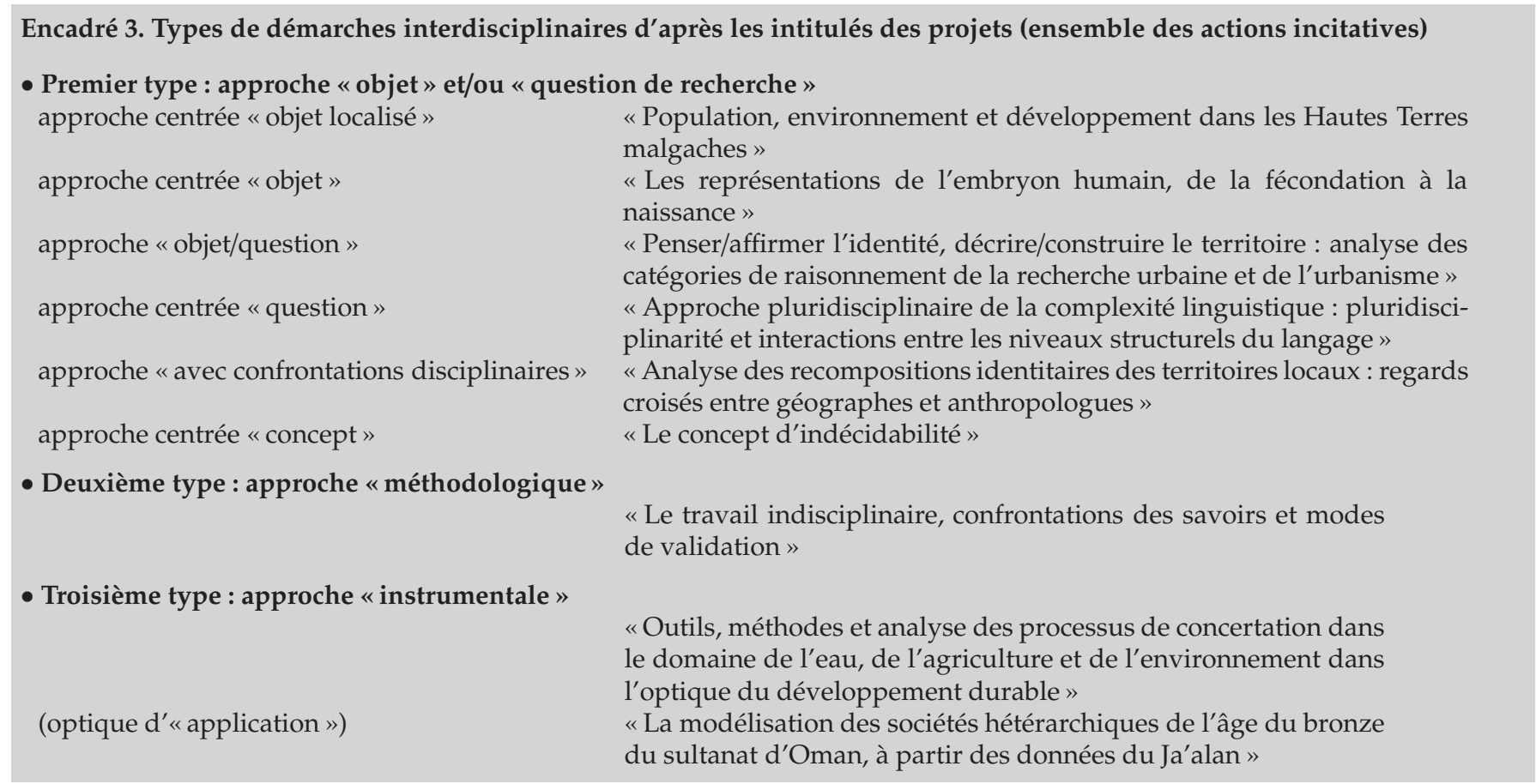

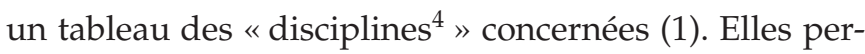
mettent en outre d'établir deux typologies : une des démarches interdisciplinaires envisagées en fonction de l'objectif scientifique poursuivi (2) et une des associations de disciplines (3).

\section{(1) Les disciplines concernées}

La forte prédominance des disciplines et spécialités de sciences humaines et sociales s'explique par le fait que l'ACI leur est explicitement destinée. Il n'en demeure pas moins que le souhait d'ouverture vers les sciences non sociales exprimé dans l'appel à propositions a trouvé un certain écho parmi les équipes. Les deux grands domaines scientifiques (sciences physiques, chimiques et de la Terre, d'une part, et sciences de la vie et sciences médicales, d'autre part) sont représentés, avec une sensible prépondérance des sciences du vivant. Les sciences de l'ingénieur figurent également dans ce partenariat. Les mathématiques sont à la fois vues comme discipline en soi et, en tant que mathématiques appliquées, comme une des disciplines de traitement des données, parmi d'autres. On doit souligner le caractère la plupart du temps très spécialisé des appellations utilisées; cela correspond aux exigences du traitement de la question précise abordée par le projet.

\footnotetext{
${ }^{4}$ Le terme est mis ici entre guillemets pour signifier que ce sont les termes utilisés par les intéressés pour s'identifier qui seront repris dans tout le texte chaque fois qu'il sera fait référence aux disciplines en cause dans les analyses.
}

\section{(2) Les démarches interdisciplinaires annoncées : une typologie par leurs objectifs}

$\mathrm{Au}$ vu des intitulés des projets, trois grands types de démarche illustrant chacun une raison d'avoir recours à l'interdisciplinarité peuvent être distingués ( $c f$. 1'Encadré3, qui donne des exemples d'intitulés de projets renvoyant à chacun des types, ainsi qu'aux sous-types).

Le premier type (approche « objet » et/ou «question de recherche ») renvoie à l'approche habituelle centrée soit « objet », soit « question ». À ceci près qu'il s'agit en l'occurrence, grâce à l'interdisciplinarité, de considérer conjointement différentes facettes de l'un ou de l'autre. Parmi les projets qui relèvent de cette première démarche, il en est qui sont d'un genre que l'on pourrait qualifier de classique. C'est l'approche dite «objet localisé ». Il s'agit d'études de cas géographiquement localisées. L'interdisciplinarité recherchée résulte de l'objectif que se donne l'équipe d'accéder à une compréhension d'ensemble d'une situation ou d'une évolution renvoyant à un terrain - au sens plein du terme - plus ou moins vaste, mais spatialement bien circonscrit.

D'autres projets sont également centrés sur un objet, mais cet «objet » n'est pas défini par une référence à une réalité géographique qu'il convient de traiter en elle-même : c'est l'approche " objet » proprement dite. À propos de ces deux cas de figure, on pourrait parler de "monographie interdisciplinaire », dans la mesure où l'analyse est centrée sur les objets eux-mêmes et où l'objectif de la recherche est de s'en donner une compréhension. 
L'objet que l'on se propose d'étudier peut, au contraire, n'être qu'un moyen de traiter d'une question de recherche et c'est en fait, à partir et au-delà de lui, celle-ci que le projet aborde et qu'il veut traiter (approche « objet/question »). C'est précisément ce qui se fait généralement dans le cadre disciplinaire, au prix donc d'un assujettissement total de l'analyse à un seul paradigme. Le recours à l'interdisciplinarité procède de l'idée selon laquelle cette réduction unilatérale tronque la compréhension de la question posée. Il en va de même des projets qui s'attaquent directement, quant à eux, à une question de recherche (approche «question »).

Ces différentes démarches sont parfois envisagées comme une occasion de dialogue de fond entre les disciplines elles-mêmes ; les projets affichent alors explicitement parmi leurs objectifs la confrontation des points de vue de celles qu'ils mobilisent, donnant par là à penser que les modalités de cette confrontation font partie de la recherche qu'ils entreprennent et que les conclusions qui en seront tirées feront donc partie de ses résultats (approche «avec confrontations disciplinaires »).

D'autres projets, enfin, se donnent comme objectif de clarifier un concept (approche "concept»). Et, là aussi, cela se fait en refusant la clôture disciplinaire dans laquelle cet exercice se fait habituellement et, en quelque sorte, par définition, le concept étant généralement conçu en fonction même de cette clôture et considéré comme l'outil par excellence de la démarche disciplinaire.

Les projets illustrant le second type de démarche se situent sur un tout autre plan. Il s'agit alors bel et bien de travailler sur la méthodologie même de l'interdisciplinarité (approche «méthodologique ») et de contribuer par là à la construction d'une démarche interdisciplinaire en tant que telle. Cela se fait en identifiant les questions scientifiques, voire épistémologiques, que la mise en pratique de cette démarche pose, et en imaginant - et en testant - des procédures destinées à les traiter.

C'est le même objectif que visent les projets de troisième type. Mais ils font, quant à eux, le choix de privilégier, pour ce faire, la voie directe de l'outil, d'un outil considéré comme susceptible en soi de lever les obstacles théoriques et épistémologiques qu'aborde le type précédent. On peut qualifier cette dernière approche $d^{\prime}$ « instrumentale ». Certains projets visent la conception d'un outil. Mais la plupart d'entre eux, au contraire, partent d'un outil existant-et éventuellement de la théorie qui va avec - et se situent plutôt dans une démarche $\mathrm{d}^{\prime}$ " application » (avec ce que cela peut supposer d'innovation, cela va sans dire). Il s'agit en somme de tester la possibilité de doter une approche disciplinaire d'outils nouveaux. En l'occurrence, l'outil en question est la plupart du temps l'informatique ou les mathématiques appliquées ${ }^{5}$. Les

\footnotetext{
${ }^{5}$ On considérera dans les lignes qui suivent ces deux spécialités comme des « disciplines».
}

projets allant dans ce sens répondent d'ailleurs à une sollicitation claire de l'appel à propositions qui, à propos du thème "Approches de la complexité", souhaitait soutenir des projets associant mathématiciens, statisticiens et informaticiens à des spécialistes de sciences humaines et sociales. Ces deux formes de démarche «instrumentale» (conception et application) peuvent être associées à celles du premier ou du second type.

Les projets de second et de troisième type sont nettement minoritaires (de l'ordre de la douzaine chacun). Les propositions s'inscrivent donc massivement dans le premier type. Parmi elles, celles qui portent sur des concepts se comptent sur les doigts d'une main et celles qui mentionnent nettement dans leur intitulé une confrontation des disciplines sont également de l'ordre de la douzaine seulement. Les propositions de nature méthodologique sont donc, au total, en nombre faible relativement à l'ensemble des réponses. Elles n'en sont pas moins de l'ordre de la cinquantaine. En outre, la confrontation entre les disciplines s'exerce au-delà de son simple affichage (qui peut d'ailleurs n'être qu'un affichage d'opportunité) ; elle résulte avant tout de la composition disciplinaire des équipes.

\section{(3) Les démarches interdisciplinaires annoncées : une typologie par les associations de disciplines}

De ce point de vue, les différents cas de figure qui apparaissent dans les propositions sont les suivants :

1) association de deux disciplines de sciences humaines et sociales ;

2) association de plus de deux disciplines de sciences humaines et sociales ;

3) association d'une ou de plusieurs disciplines de sciences humaines et sociales et de disciplines apportant une technique de recherche;

4) association d'une ou de plusieurs disciplines de sciences humaines et sociales à une ou plusieurs disciplines des sciences de la Terre, de la vie ou de l'ingénieur ;

5) même configuration que ci-dessus à laquelle s'ajoute une discipline de traitement des données.

La ventilation des propositions entre ces différentes catégories, montre que :

- comme on pouvait s'y attendre, ce sont, parmi l'ensemble des formes d'associations identifiées, les plus simples, à savoir les associations de deux ou de plus de deux disciplines de sciences humaines et sociales (et seulement de sciences humaines et sociales) qui sont, et de loin, les plus fréquentes; on notera néanmoins que les associations de plus de deux disciplines (trois la plupart du temps, mais cela peut aller jusqu'à cinq) sont presque aussi fréquentes (117) que celles, minimales, de deux disciplines (138); 
- les associations d'une ou de plusieurs disciplines de sciences humaines et sociales et de disciplines apportant une technique de recherche sont en nombre non négligeable (52); il est en outre intéressant de noter que, dans la majorité des cas, elles incluent plus d'une discipline de sciences humaines et sociales : ce sont souvent de véritables équipes interdisciplinaires qui s'associent à une discipline (voire deux) de traitement des données ;

- l'association d'une ou de plusieurs disciplines de sciences humaines et sociales à une ou plusieurs disciplines des sciences de la Terre, de la vie ou de l'ingénieur, non seulement n'est pas marginale, mais est même plus fréquente (83) que celle de la catégorie précédente; compte tenu du centrage des appels à propositions sur les sciences humaines et sociales, c'est sans doute là le constat le plus inattendu et le plus significatif de cette analyse des réponses des équipes ;

- cette forte présence d'une interdisciplinarité sortant du cadre strict des sciences humaines et sociales est encore renforcée si l'on veut bien considérer que la dernière catégorie, en dépit de son faible effectif (10), y concourt, n'étant qu'une variante sophistiquée de la précédente.

Il ne s'agit évidemment pas de la même interdisciplinarité selon que l'on a affaire, à une extrémité, à une association de deux - ou même de plus de deux - disciplines de sciences humaines et sociales ou, à l'autre extrémité, à une association de disciplines de sciences humaines et sociales et de disciplines des sciences de la Terre, de la vie ou techniques.

Dans le premier cas, la proximité peut être telle entre les disciplines associées que les appellations disciplinaires différentes font illusion quant à l'interdisciplinarité qui est réellement en jeu. On a alors affaire à une interdisciplinarité de proximité, quand ce n'est pas à une quasi-endogamie disciplinaire. Ce qui n'exclut pas que, dans certains cas, il se puisse au contraire que les disciplines associées n'aient aucune habitude de travail en commun et procèdent de points de vue fort distants les uns des autres, voire contradictoires. Par ailleurs, plus le nombre des disciplines associées est élevé, plus il y a de chances qu'il s'agisse d'une véritable interdisciplinarité. Seul un examen approfondi des propositions permettrait de voir ce qu'il en est.

Dans le cas où les sciences humaines et sociales sont associées à des disciplines d'autres domaines (Encadré4), on a affaire à une interdisciplinarité qui est tout le contraire d'une interdisciplinarité de proximité ; c'est une interdisciplinarité élargie, certaine, pour ne pas dire radicale. La question qui se pose alors est de savoir sur quelle base se fait l'association : quel est le statut respectif, dans l'association, des sciences de la Terre, de la vie ou techniques, d'une part, et des sciences humaines et sociales, d'autre part? Sont-elles les unes et les autres des partenaires à part entière ou les unes ont-elles par rapport aux autres une simple fonction d'expertise, si ce n'est d'apport d'information? En principe en tout cas, l'appel à propositions s'adressant en priorité aux sciences sociales, ce sont elles - pour une fois - qui doivent avoir le rôle-pilote dans l'association. Là aussi, la réponse suppose d'entrer dans le détail du projet. Tout ce que l'on peut dire, c'est que les propositions dans lesquelles des chercheurs se sont volontairement mis dans ce face-àface représentent, fait notable, près du quart des réponses des équipes.

Quant aux associations impliquant des disciplines fournissant une technique de traitement des données, elles traduisent pour la plupart d'entre elles la forte pénétration des mathématiques appliquées, de l'informatique et de la modélisation dans les sciences humaines et sociales. Là aussi, se pose la question du statut respectif des disciplines les unes par rapport aux autres. La façon dont les disciplines fournissant une technique de traitement des données interviennent et les conséquences de leur mise en œuvre sur les disciplines de sciences humaines et sociales constituent autant d'innovations que l'on peut mettre au compte de la forme d'interdisciplinarité particulière que ces associations produisent. Un cas de figure retient particulièrement l'attention : c'est celui dans lequel plusieurs disciplines de sciences humaines et sociales sont associées à une discipline de traitement des données. Il serait alors intéressant d'examiner le rôle que joue cette dernière dans l'établissement du dialogue entre elles et dans la coordination de leurs démarches respectives. Cette remarque vaut aussi - et à plus forte raison pour les projets associant des disciplines des sciences humaines et sociales et des disciplines des sciences de la Terre, de la vie ou de l'ingénieur à une ou des disciplines de traitement des données.

\section{Quelle place est faite à l'étude des rapports sociétés/natures?}

Les intitulés des actions incitatives thématiques (Encadré 1), d'un côté, et, en ce qui concerne l'ACI «Terrains, techniques, théories », les mots-clés qui traduisent les thèmes particuliers auxquels il est souhaité que sa problématique - par ailleurs très générale et à caractère nettement méthodologique - s'applique (Encadré 2), expriment l'idée que la Direction de la recherche du ministère (ou, plus exactement, le groupe d'experts à qui elle a confié la tâche) se faisait en 2002 des thématiques de recherche qui doivent être considérées prioritaires pour les sciences sociales en France aujourd'hui. En ce qui concerne les actions thématiques "Sociétés et cultures dans le développement durable", «Normes, pratiques et régulations des politiques publiques » et « Environnement et santé », le message est dans l'intitulé lui-même. Grâce à son caractère très général, l'ACI «Terrains, techniques, théories » présente au contraire l'intérêt de donner une image plus ouverte 


\begin{tabular}{|c|c|c|}
\hline Thème & Intitulé & Disciplines \\
\hline $\begin{array}{l}\text { Approches de la } \\
\text { complexité }\end{array}$ & $\begin{array}{l}\text { Le travail indisciplinaire : confrontations, } \\
\text { savoirs et modes de validation }\end{array}$ & $\begin{array}{l}\text { sociologie, biométrie, géologie, géochimie, } \\
\text { informatique }\end{array}$ \\
\hline $\begin{array}{l}\text { Approches de la } \\
\text { complexité }\end{array}$ & $\begin{array}{l}\text { Des gestes pour utiliser des outils : entre } \\
\text { ordre de contraintes et variations cultu- } \\
\text { relles }\end{array}$ & $\begin{array}{l}\text { psychologie, neurosciences, musicologie, } \\
\text { ethnoarchéologie, biomécanique }\end{array}$ \\
\hline $\begin{array}{l}\text { L'image en sciences } \\
\text { sociales }\end{array}$ & $\begin{array}{l}\text { Incidence d'un substrat image sur la } \\
\text { conceptualisation de l'espace et du geste } \\
\text { et sur sa verbalisation }\end{array}$ & $\begin{array}{l}\text { neurosciences, sciences et techniques des activités } \\
\text { physiques et sportives, physiologie, psychologie }\end{array}$ \\
\hline SHS des SHS & $\begin{array}{l}\text { Mesure des variables latentes en sciences } \\
\text { humaines et sociales : problèmes métho- } \\
\text { dologiques et psychométriques }\end{array}$ & $\begin{array}{l}\text { sciences de gestion, psychométrie, statistiques, } \\
\text { neurosciences }\end{array}$ \\
\hline SHS des SHS & $\begin{array}{l}\text { La notion de fonction dans les sciences } \\
\text { humaines et dans les sciences biologiques } \\
\text { et médicales }\end{array}$ & $\begin{array}{l}\text { philosophie et histoire des sciences (biologie, } \\
\text { psychologie), psychologie cognitive }\end{array}$ \\
\hline SHS des SHS & $\begin{array}{l}\text { Gouvernance du système des disciplines } \\
\text { scientifiques }\end{array}$ & $\begin{array}{l}\text { linguistique, sémiotique, biologie, sciences de la } \\
\text { cognition }\end{array}$ \\
\hline SHS des SHS & La preuve & $\begin{array}{l}\text { civilisation grecque, philosophie, éthique biomédicale, } \\
\text { études germaniques, logique, philosophie des sciences, } \\
\text { physique théorique }\end{array}$ \\
\hline $\begin{array}{l}\text { Règles, normes, } \\
\text { régularités }\end{array}$ & Les règles déontologiques & $\begin{array}{l}\text { droit public, hématologie, sciences de gestion, } \\
\text { droit privé }\end{array}$ \\
\hline La peur et les risques & $\begin{array}{l}\text { La peur et le cerveau : approche psycho- } \\
\text { cognitive par imagerie et par résonance } \\
\text { magnétique fonctionnelle }\end{array}$ & $\begin{array}{l}\text { neurosciences, musicothérapie, psychologie sociale, } \\
\text { musicologie, psychologie }\end{array}$ \\
\hline $\begin{array}{l}\text { Le naturel et } \\
\text { l'artificiel }\end{array}$ & $\begin{array}{l}\text { Nature et artifice à l'épreuve des nou- } \\
\text { velles technologies }\end{array}$ & philosophie, sociologie, biologie \\
\hline $\begin{array}{l}\text { Pratiques et } \\
\text { techniques du corps }\end{array}$ & $\begin{array}{l}\text { Langage tactile et orientation du corps } \\
\text { dans l'espace : une assistance technique } \\
\text { au contrôle de l'orientation dans l'espace } \\
\text { tridimensionnel dans le cas de déficits } \\
\text { sensorimoteurs }\end{array}$ & $\begin{array}{l}\text { modélisation en sciences cognitives et sociales, } \\
\text { informatique, biophysique, ingénierie cognitive, } \\
\text { neurosciences, psychologie }\end{array}$ \\
\hline $\begin{array}{l}\text { Traduire, interpréter, } \\
\text { transmettre }\end{array}$ & $\begin{array}{l}\text { Les sciences et les arts à l'épreuve de } \\
\text { l'interprétation et de la traduction : dia- } \\
\text { logue interdisciplinaire et création choré- } \\
\text { graphique dans le cadre de la transmis- } \\
\text { sion des savoirs }\end{array}$ & $\begin{array}{l}\text { ethnologie, sociologie, physique nucléaire, biologie, } \\
\text { danse }\end{array}$ \\
\hline $\begin{array}{l}\text { Traduire, interpréter, } \\
\text { transmettre }\end{array}$ & $\begin{array}{l}\text { Tradition/traduction/transmission: la tra- } \\
\text { duction «SHS-Sciences dures » dans tous } \\
\text { ses états }\end{array}$ & $\begin{array}{l}\text { philosophie, musicologie, mathématiques, physique } \\
\text { théorique, astrophysique }\end{array}$ \\
\hline $\begin{array}{l}\text { Traduire, interpréter, } \\
\text { transmettre }\end{array}$ & $\begin{array}{l}\text { Réflexions sur des pratiques de traduc- } \\
\text { tion, d'interprétation et de transmission } \\
\text { des savoirs dans le domaine du dévelop- } \\
\text { pement durable }\end{array}$ & $\begin{array}{l}\text { sociologie et économie du développement, sciences de } \\
\text { l'éducation, informatique, biologie, chimie, sciences du } \\
\text { langage }\end{array}$ \\
\hline
\end{tabular}

de ces centres d'intérêt. On ne sera pas surpris de trouver parmi eux la communication sous toutes ses formes ( "Traduire, interpréter, transmettre »), et plus particulièrement la place de l'image dans les sociétés («De l'icône au visuel : 1'image en sciences humaines et sociales »), les identités sociales : spatiales, culturelles, professionnelles, politiques, sexuelles («Identité(s)»), la mondialisation («Monde(s), mondialisation »), les âges de la vie (« Âges, temps de vie et rapport de génération »), et d'y retrouver les normes sociales («Règles, normes, régularités»). Ce sont là, en effet, des questions de société contemporaines majeures.
Rien d'étonnant, donc, à ce que l'on trouve également, de façon explicite, aux côtés de ces thèmes, ceux du rapport au naturel («Le naturel et l'artificiel»), aux risques («La peur et les risques »), au corps et en particulier à la santé («Pratiques et techniques du corps»). Autant de questions qui sont aussi des questions de société actuelles et qui traitent d'une manière ou d'une autre les rapports sociétés/natures. Et c'est bien, en effet, de quoi traitent dans leur quasi-totalité les propositions classées dans ces rubriques. Mais l'examen des listes des propositions montre qu'environ un tiers, si ce n'est la moitié, des propositions classées sous les autres mots-clés de 
l'ACI sont dans le même cas. La seule exception notable concerne le mot-clé «Âge, temps de la vie, rapport de génération ». Les intitulés des propositions mettent en évidence des recoupements thématiques nets avec l'ACI «Sociétés et cultures dans le développement durable » et l'ACT « Environnement et santé », mais aussi avec l'ACI «Normes, pratiques et régulations des politiques publiques ». En effet, si celle-ci est beaucoup plus générale, elle n'en inclut pas moins nécessairement les politiques en rapport avec le vivant, l'environnement, la santé. De fait, un quart des propositions des équipes s'avèrent porter directement sur des questions ayant trait à ces politiques ; par ailleurs, plusieurs autres propositions issues de ce même appel abordent des problèmes de l'action publique qui ont une portée générale : au total, ce sont donc les deux tiers des propositions qui peuvent être considérées comme présentant un intérêt pour la compréhension de ces politiques.

Un autre constat retient l'attention dans ces décomptes; il concerne l'ACI «Terrains, techniques, théories » : c'est la forte place que les questions sur l'interdisciplinarité soulevées par l'analyse des rapports sociétés/natures occupent dans l'ensemble des propositions d'ordre méthodologique. Ce sont alors, en fait, les rapports sciences/sociétés/natures qui sont en cause, car la question posée est celle de savoir comment inclure les rapports entre disciplines (i.e. entre sciences) dans les rapports sociétés/natures. Ceci vaut en particulier pour les propositions qui sont classées sous les mots-clés «Approches de la complexité », "SHS des SHS » (commun, rappelons-le, aux quatre appels), «Mesures, données : catégorisation, agrégation, modélisation, numérisation, diffusion » et «L'erreur » : les deux tiers de ces propositions au minimum y renvoient. Mais on doit également noter qu'un quart des propositions classées sous le motclé «Traduire, interpréter, transmettre » peuvent également être retenues à ce titre.

Au total, une bonne moitié des projets soumis par les équipes en réponse à l'ACI " Terrains, techniques, théories " abordent l'analyse des rapports sociétés/natures, que ce soit à travers des thèmes et des questions de recherche ayant trait à l'environnement, au vivant, au corps et à la santé ou à propos des questions de méthode concernant l'interdisciplinarité que leur analyse requiert. Si l'on ajoute à ce contingent de réponses la totalité de celles faites à l'ACI «Sociétés et cultures dans le développement durable » et à l'ACT «Environnement et santé », ainsi que les deux tiers de celles faites à l'ACI «Normes, pratiques et régulations des politiques publiques », ce sont largement les deux tiers des réponses à l'ensemble de ces actions qui sont dans ce cas. Ce décompte illustre la forte prégnance de ces thématiques de recherche. On peut également en conclure que les analyses faites ci-dessus sur l'ensemble du corpus des réponses reflète les formes de l'interdisciplinarité qui sont mises en œuvre dans ce sous-ensemble et les démarches méthodologiques qui y sont développées à propos de l'interdisciplinarité.

L'examen des intitulés des propositions qui entrent dans ce sous-ensemble est extrêmement instructif. Celles qui s'inscrivent dans le cadre de l'ACI "Sociétés et cultures dans le développement durable » et de l'ACT «Environnement et santé » abordent les thèmes de recherche habituels s'agissant de ces deux grandes questions. Le choix de consacrer à ces dernières des actions d'incitation particulières traduit d'abord la volonté de les désigner à l'attention comme prioritaires et le souci de dynamiser les recherches les concernant - ce qui peut tout à fait se justifier compte tenu du faible engagement actuel des sciences sociales dans ces domaines. En outre, dans le cas de l'ACI « Sociétés et cultures dans le développement durable», cela se fait, on l'a vu, en incitant à développer l'interdisciplinarité. Et cela se traduit effectivement dans les propositions de recherche. Si innovation il doit y avoir, elle est là. À ce propos, il convient de souligner qu'en dépit de l'absence de recommandation en ce sens dans l'appel à propositions de l'ACT «Environnement et santé », 11 des 26 réponses reçues comportent une association de disciplines et, dans 8 d'entre elles, les sciences sociales sont associées à des disciplines des sciences de la vie. L'envie d'interdisciplinarité s'est imposée en dehors de toute contrainte.

La philosophie de l'ACI «Terrains, techniques, théories » est tout autre. L'appel à la réflexivité méthodologique, théorique et épistémologique que contient son intitulé, son inscription franche et argumentée dans la démarche interdisciplinaire, le caractère nettement méthodologique de certains des thèmes affichés et celui très transversal par rapport aux disciplines des autres sont autant d'invitations à l'innovation. Ceci se ressent dans l'originalité d'un grand nombre des propositions, si l'on en juge par leurs intitulés et par les montages interdisciplinaires inédits auxquels elles donnent lieu (Encadré 4). Une bonne partie de ces propositions porte sur les rapports entre disciplines, que ceux-ci soient abordés en tant que tels ou à travers un des thèmes affichés. Et les sciences sociales ne sont pas les seules concernées. C'est là un constat qui mérite attention : l'initiative prise en lançant cette $\mathrm{ACI}$ révèle bien que la réflexion sur les bases de la division du travail scientifique, prélude à la quête d'une nouvelle pratique de la recherche, ne demande qu'à s'exprimer.

Toutes ces analyses souffrent d'une sérieuse limite : ne s'appuyant que sur les intitulés des projets de recherche et sur la liste des disciplines qui sont censées y être associées, elles s'en tiennent à leur façade. Ceci peut, à vrai dire, n'avoir guère d'importance. Il en est ainsi quand les disciplines associées sont très proches et quand elles s'associent pour traiter d'un objet ou d'une question qu'elles partagent de longue date. Dans ce cas, aucune innovation dans la démarche n'est 
nécessaire. C'est souvent le cas lorsque l'association interdisciplinaire ne porte que sur deux disciplines de sciences sociales. Mais cette limite devient gênante lorsque l'on dépasse le stade de ce qui est, au mieux, une interdisciplinarité de routine. Il est alors impossible de déceler la part de l'effet d'affichage que peut éventuellement contenir la présentation du projet à travers son intitulé et la composition de l'équipe interdisciplinaire censée le porter; partant, il est tout autant impossible de se rendre compte de la réalité de l'effort qui sera tenté pour atteindre l'objectif annoncé. Et surtout, cela empêche de découvrir ce qui est le cœur du projet, à savoir la démarche de recherche qu'il est proposé $\mathrm{d}^{\prime} \mathrm{y}$ suivre dans ce but. Or, c'est dans celle-ci que réside l'innovation impliquée par l'interdisciplinarité. Il ne suffit pas, en effet, de juxtaposer des disciplines, il faut se donner une méthode pour les faire travailler de concert.

Cela dit, aussi superficielles soient-elles, les analyses qui viennent d'être faites permettent au moins de subodorer la part d'innovation requise pour que les associations de disciplines annoncées honorent l'engagement qu'elles prennent en se donnant les objectifs de recherche partagés qu'elles affichent. Et il ressort clairement que, dans la très grande majorité des cas, cette part d'innovation est importante.

Reste à mesurer le degré d'engagement des équipes postulantes dans cette voie. Les résultats des projets de recherche qui ont été sélectionnés suite à ces actions incitatives donneront sans doute un début de réponse.

To access this journal online: www.edpsciences.org 